\title{
WELL DISTRIBUTED SEQUENCES OF INTEGERS
}

\author{
BY \\ WILLIAM A. $\operatorname{VEECH}\left({ }^{1}\right)$
}

\begin{abstract}
Niven's notion of a uniformly distributed sequence of integers is generalized to well distribution, and two classes of integer sequences are studied in terms of this generalization.
\end{abstract}

1. Introduction. Niven [5] defines a sequence of integers $q_{1}, q_{2}, \ldots$ to be "uniformly distributed" if it satisfies a condition equivalent to the following: for every nonintegral rational number $\alpha$ the relation

$$
\lim _{N \rightarrow \infty} \frac{1}{N} \sum_{n=1}^{N} e\left(q_{n} \alpha\right)=0
$$

holds, where $e(y)=\exp (2 \pi i y)$. We use the same terminology but reserve it for those sequences satisfying (1) for all nonintegral real numbers $\alpha$. Then we say $q_{1}, q_{2}, \ldots$ is well distributed if it satisfies the stronger requirement that for every nonintegral real number $\alpha$

$$
\lim _{N \rightarrow \infty} \frac{1}{N} \sum_{n=1}^{N} e\left(q_{n+k} \alpha\right)=0
$$

holds uniformly for $k \geqq 0$.

We now state two theorems concerning well distribution. The first is proved in $\S 2$ and the second in $\S 3 .[\cdot]$ is the greatest integer function.

THEOREM 1. Let $P(n)=a_{0}+a_{1} n+\cdots+a_{t} n^{t}$ be a polynomial with real coefficients, and define $q_{n}=[P(n)]$ for each $n$. The following statements are pairwise equivalent:

(i) $q_{1}, q_{2}, \ldots$ is well distributed.

(ii) $q_{1}, q_{2}, \ldots$ is uniformly distributed.

(iii) Either $t=1$ and $a_{1}=1 / q$ for some integer $q$, or else $t \geqq 2$ and $a_{1}, \ldots, a_{t}$ do not lie in a singly generated additive subgroup of the reals.

REMARK. If $x$ and $y$ are real numbers with $x$ irrational Niven shows $q_{n}=[n x+y]$ is uniformly distributed by his definition. The same sequence is not uniformly distributed in the sense of the present note. The limits (1) do all exist, but for certain (necessarily irrational) $\alpha$, for example, $\alpha=1 / x$, they are not 0 .

In preparation of Theorem 2 we fix an irrational real number $\theta$ with continued fraction expansion $\theta=\left[a_{0} ; a_{1}, a_{2}, \ldots\right]$. Recall that $\theta$ has bounded partial quotients

Received by the editors September 22, 1970.

AMS 1970 subject classifications. Primary 10F40.

Key words and phrases. Uniformly distributed, well distributed, bounded partial quotients. (') Research supported by NSF grant GP-18961.

Copyright (C) 1971, American Mathematical Society 
if $\sup _{n} a_{n}<\infty$. Let $I \subseteq[0,1)$ be an interval, $I=(\beta, \gamma)$, subject only to the restriction that $\gamma-\beta$ is not congruent to an integral multiple of $\theta$ modulo 1 . Now a sequence $S(\theta, I)=q_{1}, q_{2}, \ldots$ is defined by letting $q_{n}$ be the number of $j, 1 \leqq j \leqq n$, such that $j \theta \in I$ (modulo 1).

THEOREM 2. A necessary and sufficient condition for $S(\theta, I)$ to be well distributed for all intervals $I$, as restricted above, is that $\theta$ have bounded partial quotients.

REMARK. We have only to prove the sufficiency in Theorem 2 since necessity is contained in the results of [7]. In fact, if $\theta$ has unbounded partial quotients, there exists an uncountable set of $t \in(0,1)$ and for every $t$ in this set an uncountable number of intervals of length $t$ such that the limit (1) does not even exist for $S(\theta, I)$ and $\alpha=\frac{1}{2}$.

REMARK. Despite the preceding remark, there are statements valid for arbitrary irrationals. For example, (A) for almost all $t \in(0,1)$ (depending on $\theta$ ) if $I$ has length $t, S(\theta, I)$ is well distributed, and (B) if $t$ is rational, and if $I$ has length $t$, then $S(\theta, I)$ is well distributed for every irrational number $\theta$.

2. Proof of Theorem 1. Since (ii) is weaker than (i) we have (i) $\Rightarrow$ (ii). We now prove the contrapositive of (ii) $\Rightarrow$ (iii). Thus, we suppose $a_{1}, \ldots, a_{t}$ are all integral multiples of a fixed number $\theta$, and if $t=1, a_{1}$ does not have the form $1 / q, q \in Z$. We may clearly assume $t \geqq 1$, since there is nothing to prove if $t=0$. It is necessary to treat separately the cases $\theta$ rational and $\theta$ irrational.

Case 1. $\theta$ rational. We write $P(n)=a_{0}+(1 / q)\left(b_{1} n+\cdots+b_{t} t^{t}\right)$, where $q, b_{1}$, $\ldots, b_{t} \in Z$, and if $t=1, b_{1} \neq \pm 1$. Define, for $0 \leqq r<q, P_{r} \in Z[x]$ and $\lambda_{r} \in Z$ by

$$
P_{r}(n)=P(q n+r)-P(r), \quad \lambda_{r}=[P(r)] .
$$

We have $[P(q n+r)]=P_{r}(n)+\lambda_{r}$ for all $n$ and $r$. Define $Q_{r}(n)=P_{r}(n)+\lambda_{r}$, also in $Z[x]$.

Let $p>1$ be an integer, and define $\alpha_{v}, \nu=1, \ldots, p-1$, by $\alpha_{\nu}=\nu / p$. If (1) is to hold for $q_{n}=[P(n)]$ and $\alpha=\alpha_{v}$, then because the sequence $e\left(\alpha_{v} q_{n}\right)$ has period $p q$, we have

$$
\sum_{n=0}^{p-1} \sum_{r=0}^{q-1} e\left(\alpha_{v} Q_{r}(n)\right)=\sum_{n=0}^{p q-1} e\left(\alpha_{v}[P(n)]\right)=0 .
$$

Let $\tau_{j}, 0 \leqq j<p$, be the number of pairs $(r, n), 0 \leqq r<q, 0 \leqq n<p$, such that $e\left((1 / p) Q_{r}(n)\right)=e(j / p)$. Then (2) is the same as

$$
\sum_{j=0}^{p-1} \tau_{j} e\left(\alpha_{v} j\right)=0 \quad(\nu=1, \ldots, p-1) .
$$

This implies $\tau_{j}=\tau$ is independent of $j$, and since $\tau_{0}+\cdots+\tau_{p-1}=p q$, we have $\tau=q$. A consequence of the lemma to follow is that there exist primes $p$ and integers $l$ such that each $Q_{r}-l$ has $t$ zeros modulo $p$. Thus $t q=q$, or $t=1$. Suppose for the moment this has been proved. Then $P(n)=a_{0}+(b / q) n, b=b_{1} \neq \pm 1$, and $Q_{r}(n)$ $=b n+\lambda_{r}$ for each $r$. We may suppose $(b, q)=1$. Let $p>1$ be a divisor of $b$. If 
$\alpha_{\nu}=v / p$, as above, then by (2) $p \sum_{n=0}^{q-1} e\left(\alpha_{\nu} \lambda_{r}\right)=0$. Let $\tau_{j}, 0 \leqq j<p$, be the number of $r$ such that $e\left(\lambda_{r} / p\right)=e(j / p)$. Then as above $\tau_{j}=\tau$ and $\tau p=q$. Thus, $p \mid q$, and $(b, q) \neq 1$, a contradiction.

The following lemma is probably well known. Our original proof used an argument in Theorem 9 of [4], however M. Fried has pointed out to us a very simple proof. We use Fried's argument below.

LEMma 1. Let $G_{0}, \ldots, G_{q-1} \in Z[x]$ be irreducible and have degrees $d_{0}, \ldots, d_{q-1}$ $\geqq 1$, respectively. There exist infinitely many primes p such that for each $r, 0 \leqq r \leqq q-1$, $G_{r}$ has $d_{r}$ distinct zeros modulo $p$.

REMARK. To apply the lemma in the above, simply choose any integer $l$ such that $G_{r}=Q_{r}-l$ is irreducible, $0 \leqq r \leqq q-1$.

Proof. Let $S$ be the set of primes which divide neither the discriminant nor the lead coefficient of any of the $G_{i}$ 's. $S$ contains almost all primes. If $p \in S$, and if $G_{r}$ splits completely modulo $p$, then $G_{r}$ has $d_{r}$ distinct zeros modulo $p$. Let $M$ be a normal splitting field for $\left\{G_{r} \mid 0 \leqq r \leqq q-1\right\}$, and let $\alpha$ be a primitive element for $M$ with minimal polynomial $f \in Z[x]$. Now any zero of $G_{r}$ can be expressed as a rational function of any zero of $f$, and any zero of $f$ can be expressed as a rational function of any other zero of $f$. Using this fact one sees readily that by discarding an additional finite set of primes from $S$ we obtain a set $S_{0}$ containing almost all primes with the property that if $p \in S_{0}$ and if $f$ has one zero modulo $p$, then each $G_{r}($ and $f$ ) split completely modulo $p$. We are reduced to proving $f$ has one zero modulo $p$ for an infinite number of primes $p$. Indeed, if $f(n)$ is representable in terms of $p_{1}, \ldots, p_{r}$ for all $n$, then $f\left(n+\left(\prod_{i=1}^{r} p_{i}\right)^{s}\right)=f(n)+\lambda_{s} p_{1}^{s} \cdots p_{r}^{s}=p_{1}^{l_{1}} \cdots p_{r}^{l_{r}}$ for all $s, l_{1}, \ldots, l_{r}$ depending on $n$ and $s$. This is a contradiction for any $n$ such that $f(n) \neq 0$ if $s$ is sufficiently large. The lemma is proved.

Case 2. $\theta$ irrational. Let $\lambda_{n}$ be defined by

$$
P(n)=[P(n)]+\lambda_{n} \quad\left(0 \leqq \lambda_{n}<1\right) .
$$

Any nonzero element among $a_{1}, \ldots, a_{t}$ is a multiple of $\theta$ and hence irrational. Therefore, by Weyl's theorem [9] on the uniform distribution of the fractional parts of polynomials having at least one coefficient of order greater than 0 irrational, the sequence $\lambda_{1}, \lambda_{2}, \ldots$ is uniformly distributed on the unit interval. Also, since $a_{j} \theta^{-1} \in Z, 1 \leqq j \leqq t$, we have $P(n) \theta^{-1} \equiv a_{0} \theta^{-1}$ (modulo 1) for all $n$. Therefore, by (3) $[P(n)] \theta^{-1} \equiv a_{0} \theta^{-1}-\lambda_{n} \theta^{-1}$. The function $f(\lambda)=e\left(a_{0} \theta^{-1}-\lambda \theta^{-1}\right)$ is continuous on the unit interval and has nonzero integral. By the uniform distribution of $\lambda_{n}$, the limit (1) exists and is nonzero for $q_{n}=[P(n)]$ and $\alpha=\theta^{-1}$. Thus, $q_{1}, q_{2}, \ldots$ is not uniformly distributed.

Finally, we must prove (iii) $\Rightarrow$ (i). Thus, we suppose $a_{1}, \ldots, a_{t}$ do not lie in a singly generated additive subgroup of the reals. At least one of $a_{1}, \ldots, a_{t}$ is irrational, and therefore Niven's argument (used for $[n x+y]$ ) together with Weyl's 
theorem tells us $\left(1^{\prime}\right)$ holds for $q_{n}=[P(n)]$ and $\alpha$ rational. Now suppose $\alpha$ is irrational. We define $\lambda_{n}, \lambda_{n}^{\prime}, 0 \leqq \lambda_{n}, \lambda_{n}^{\prime}<1$, using (3) and

$$
P(n) \alpha=[P(n) \alpha]+\lambda_{n}^{\prime} .
$$

If $i$ and $j$ are integers, not both 0 , our assumption on $a_{1}, \ldots, a_{t}$ implies that at least one of $(i+j \alpha) a_{s}, 1 \leqq s \leqq t$, is irrational. Using Weyl's theorem and the fact $\left(i \lambda_{n}+j \lambda_{n}^{\prime}\right)$ $\equiv(i+j \alpha) P(n)(\bmod 1)$, we obtain

$$
\lim _{N \rightarrow \infty} \frac{1}{N} \sum_{n=1}^{N} e\left(i \lambda_{n+k}+j \lambda_{n+k}^{\prime}\right)=0
$$

uniformly in $k \geqq 0$. (The uniformity in $k$ was not given by Weyl but is a well-known and easy consequence of his method.) Since (5) holds for all $i$ and $j$ such that not both are 0 , Weyl's criterion, applied to the two torus, tells us the sequence $\left(\lambda_{n}, \lambda_{n}^{\prime}\right)$ is well distributed in the unit square of the $\left(\lambda, \lambda^{\prime}\right)$ plane. The function $f\left(\lambda, \lambda^{\prime}\right)$ $=e\left(\lambda^{\prime}-\alpha \lambda\right)$ is continuous on this square and has Riemann integral 0 over it. Using (3) and (4) we have $[P(n)] \alpha \equiv \lambda_{n}^{\prime}-\lambda_{n} \alpha$, and so the well distribution of $\left(\lambda_{n}, \lambda_{n}^{\prime}\right)$ implies ( $\left(1^{\prime}\right)$ for $q_{n}=[P(n)]$ and our $\alpha$. The case $t=1, a_{1}=1 / q$ being trivial, this completes the proof of Theorem 1 .

3. Proof of Theorem 2. We begin with some notation and review of known facts. Let $X$ be the compact group of real numbers modulo 1, usually to be represented as $X=[0,1)$, and let $\theta \in X$ be an irrational number, fixed for the discussion. We define $T: X \rightarrow X$ by $T x=x+\theta$. Let $\Omega=(X, \mathscr{B}, d y)$, where $\mathscr{B}$ is the Lebesgue field and $d y$ is Lebesgue measure. If $h \in \mathscr{C}$, where $\mathscr{C}$ is the space of Riemann integrable functions on $X$, we have by the Kronecker-Weyl theorem that

$$
\lim _{N \rightarrow \infty} \frac{1}{N} \sum_{n=1}^{N} h\left(T x^{n+k}\right)=\int_{X} h d y
$$

holds uniformly in $k$ and $x$. This says in particular that each $x \in X$ is " $(\Omega, T, \mathscr{C})$ strictly generic" in the terminology of [6]. Given $f \in \mathscr{C}$ with absolute value 1 everywhere, define $f^{(n)}(x), n \geqq 1$, by $f^{(1)}(x)=f(x), f^{(n+1)}(x)=f(x+n \theta) f^{(n)}(x)$. Lemma 1 of [6] asserts that the relation

$$
\lim _{N \rightarrow \infty} \frac{1}{N} \sum_{n=1}^{N} f^{(n+k)}(x)=0
$$

fails to hold uniformly in $k \geqq 0$ for some $x$ only if the equation

$$
g(y+\theta)=f(y) g(y)
$$

has a nontrivial measurable solution.

Now let $I \subseteq X$ be a set whose characteristic function is Riemann integrable. If $\alpha$ is a nonintegral real number, fixed in what follows, we define $f$ on $X$ by

$$
\begin{aligned}
f(x) & =e(\alpha), & & x \in I, \\
& =1, & & x \notin I .
\end{aligned}
$$


Then $f^{(n)}(x)=f(x) f(x+\theta) \cdots f(x+(n-1) \theta)=e\left(\lambda_{n} \alpha\right)$, where $\lambda_{n}=\lambda_{n}(x)$ is the number of $j, 0 \leqq j \leqq n-1$, such that $x+j \theta \in I$. If $I=(\beta, \gamma), 0 \leqq \beta<\gamma \leqq 1$, and if $S(\theta, I)$ $=q_{1}, q_{2}, \ldots$, then $f^{(n)}(0)=e\left(q_{n-1} \alpha\right), n \geqq 2$. Thus, Theorem 2 is reduced to proving that if $\theta$ has bounded partial quotients, and if $I=(\beta, \gamma)$ is such that $\gamma-\beta \neq j \theta$ for all $j$, then (7) can have no nontrivial measurable solution.

REMARK. If (7) has a solution for one $I$, then it has a solution for every translate of $I$. We may therefore fix notations and always assume $I=[0, t), 0<t<1$.

If $g$ is a solution to (7), then $|g(y+\theta)|=|g(y)|$, a.e. $d y$, and therefore $|g(\cdot)|$ is essentially constant. If $g$ is nontrivial, we may and shall normalize and take the constant to be 1 . Using $z^{*}$ for the complex conjugate of $z$, we rewrite (7) in the form

$$
g(x) g^{*}(x+\theta)=f(x)
$$

with its consequence

$$
g(x) g^{*}(x+n \theta)=f^{(n)}(x) .
$$

Define $\alpha_{n}=\int_{X} f^{(n)}(y) d y$. Using $g g^{*}=1$ and the continuity of translation in $L^{2}$, we conclude from (8) that

$$
\lim _{n \rightarrow 0} \alpha_{n}=1
$$

In fact, the argument on p. 9 of [7] shows (9) is also sufficient for the solvability of (7). In what follows we use only

$$
\lim _{n \theta \rightarrow 0}\left|\alpha_{n}\right|=1 .
$$

If $n>0$, the discontinuities of $f^{(n)}$ in $X$ occur at the distinct points $0,-\theta, \ldots$, $(1-n) \theta, t, t-\theta, \ldots, t+(1-n) \theta$ using our assumption $t \neq j \theta$. Between discontinuities $f^{(n)}$ is constant. If we write $X=[0,1)$ and fix a discontinuity $y=-k \theta$, then $f\left(y^{+}\right)$ $=e(-\alpha) f\left(y^{-}\right)$, where the + and - denote right and left limit. If $y=t-k \theta$, then $f\left(y^{+}\right)=e(\alpha) f\left(y^{-}\right)$. Assume as we may that $0<\alpha<1$, and let $\alpha_{0}=\min (\alpha, 1-\alpha)$. The foregoing observation implies for any point $\zeta$ on the unit circle and any successive intervals $J, J^{\prime}$ of constancy for $f^{(n)}$, that $\left|f^{(n)}(x)-\zeta\right| \geqq(\sqrt{ } 2 / 2)\left(1-\cos 2 \pi \alpha_{0}\right)^{1 / 2}=\tau$ on one of $J, J^{\prime}$. Thus, on $n$ of the $2 n$ intervals of constancy, $\left|f^{(n)}-\zeta\right| \geqq \tau$. Let $\varepsilon_{n}$ be for each $n$ the length of the shortest interval of constancy. Using (10) and the observation just made, we have

$$
\lim _{n \theta \rightarrow 0} n \varepsilon_{n}=0 .
$$

REMARK. The argument on p. 15 of [7] shows the set of $t$ for which (11) holds has measure 0 , and this does not depend on $\theta$ having bounded partial quotients. We have for almost all $t \in(0,1)$ that if $I=(\beta, \gamma), \gamma-\beta=t$, then $S(\theta, I)$ is well distributed. 
If $0<s<1$, we define $\varphi_{s}, \psi_{s}$ on $X$ by

$$
\begin{aligned}
\varphi_{s}(x) & =e(\alpha), & & 0 \leqq x<s, \\
& =1, & & s \leqq x<1, \\
\psi_{s}(x) & =e(-\alpha), & & 0 \leqq x<s, \quad\left(=\varphi_{s}^{*}(x)\right) . \\
& =1, & & s \leqq x<1,
\end{aligned}
$$

If $n>0$, define

$$
\varphi^{(n)}=\varphi_{-\theta} \varphi_{-2 \theta} \cdots \varphi_{(1-n) \theta} \psi_{t} \psi_{t-\theta} \cdots \psi_{t+(1-n) \theta},
$$

the subscripts, as usual, being taken modulo 1 . We note that $\varphi^{(n)}$ and $f^{(n)}$ possess the same discontinuities with the same jumps, and therefore $\varphi^{(n)}=e(-\alpha) f^{(n)}(0)^{*} f^{(n)}$ (compare the values at $x=0$ ). Letting $\beta_{n}=\int_{X} \varphi^{(n)} d y,(10)$ implies

$$
\lim _{n \rightarrow \rightarrow 0}\left|\beta_{n}\right|=1 \text {. }
$$

For each $n>0$ define $z_{n}$ and $j=j_{n},|j|<n$, by $\|j \theta-t\|=\min _{|i|<n}\|i \theta-t\|=z_{n}(\|\cdot\|$ denotes distance to nearest integer). $j_{n}$ is well defined unless $2 t=(2 m+1) \theta$, and here we simply make a choice. Define $\psi^{(n)}$ by

$$
\psi^{(n)}=\varphi_{(j-n) \theta} \varphi_{(j-1-n) \theta} \cdots \varphi_{(1-n) \theta} \psi_{j \theta} \cdots \psi_{\theta}
$$

if $j=j_{n}>0$ and

$$
\psi^{(n)}=\varphi_{-\theta} \varphi_{-2 \theta} \cdots \varphi_{j \theta} \psi_{(-1-n) \theta} \cdots \psi_{(j-n) \theta}
$$

if $j<0$. In (13) the relation $\varphi_{s} \psi_{s}=1$ and the triangle inequality for the $L^{1}$ norm imply

$$
\begin{aligned}
\left\|\varphi^{(n)}-\psi_{t-j \theta} \psi^{(n)}\right\|_{1} & =\left\|\varphi^{(n)}-\psi_{t-j \theta} \psi^{(n)} \varphi_{-\theta} \cdots \varphi_{(j+1-n) \theta} \psi_{-\theta} \cdots \psi_{(j+1-n) \theta}\right\|_{1} \\
& \leqq \sum_{i=0: i \neq j}^{n-1}\left\|\psi_{t-i \theta}-\psi_{(j-i) \theta}\right\|_{1} \leqq 2(n-1) z_{n}
\end{aligned}
$$

provided 0 does not separate any of the pairs $t-i \theta,(j-i) \theta$. (By "separate" it is meant that 0 lies in the smallest component of the complement of the pair.) Define $\Lambda$ to be the set of $n$ for which $\varepsilon_{n}=z_{n}$. For these $n$ (15) applies, and so by (11) and (12) we have $\lim _{n \theta \rightarrow 0 ; n \in \Lambda}\left|\gamma_{n}\right|=1$ where $\gamma_{n}=\int_{X} \psi^{(n)} d y$. (A relation similar to (15) holds for $j_{n}<0$.)

In the above set $\mu=\operatorname{sgn} j_{n}$ and unify notation by setting

$$
\psi^{(n)}=\varphi_{\mu \theta} \varphi_{2 \mu \theta} \cdots \varphi_{j \theta} \psi_{(\mu-n) \theta} \cdots \psi_{(j-n) \theta}
$$

Should it be the case that $\|n \theta\|<\min _{1 \leqq i \leqq \mu j}\|i \theta\|, j=j_{n}$, then by pairing terms $\varphi_{\mu \theta} \psi_{(\mu-n) \theta}, \ldots, \varphi_{j \theta} \psi_{(j-n) \theta}$ we find that $\psi^{(n)}$ is 1 on a collection of $\mu j$ intervals whose total measure is $1-\mu j\|n \theta\|$ and $e(A), A=\alpha$ or $-\alpha$ always the same, on intervals totalling $\mu j\|n \theta\|$. Then $\gamma_{n}=\left(1-\mu_{j}\|n \theta\|\right)+\mu_{j}\|\mu \theta\| e(A)$ is a convex combination of 1 and $e(A)$. It follows that there exists a constant $\lambda>0$ depending on $\alpha$ such that if $\left|\gamma_{n}\right|>1-\varepsilon$ then

$$
\mu j\|n \theta\|<\lambda \varepsilon \text { or } \mu j\|n \theta\|>1-\lambda \varepsilon \text {. }
$$


Theorem 2 now follows from the argument of Proposition 7 of [7]. Since it is so short we repeat it here.

Let $p_{n} / q_{n}, n=1,2, \ldots$, be the sequence of convergents to $\theta$, and recall the inequalities

$$
1 / 2 q_{n+1}<\left\|q_{n} \theta\right\|<1 / q_{n+1}
$$

valid for all $n$. (See [3].) Since $\theta$ has bounded partial quotients, there exists $c$, $0<c<\frac{1}{2}$, such that

$$
\left\|q_{n} \theta\right\|>c / q_{n}
$$

for all $n$. Using (18) and (19) we have for all $m \geqq 0$

$$
q_{n}\left\|q_{n+m} \theta\right\|>c^{m+1} .
$$

Let $\lambda$ be chosen as in (17), and choose $\varepsilon>0$ so that $\varepsilon, \lambda \varepsilon<c^{3}$. We write $\varepsilon_{n}, z_{n}, \gamma_{n}$ for the corresponding quantities with subscripts $q_{n}$. Of course $q_{n} \theta \rightarrow 0$, and because of (18) and (11) $\varepsilon_{n}=z_{n}$ for all large $n$. Let $n_{0}$ be such that if $n \geqq n_{0}$, then $\left|\gamma_{n}\right|>1-\varepsilon$ and $q_{n} \varepsilon_{n}<\varepsilon / 2$. Letting $z_{n+1}=\|j \theta-t\|$, it will be true infinitely often that $q_{n}<|j|$ $<q_{n+1}$, and we assume $n$ has been so chosen. If also $z_{n+2}=\|j \theta-t\|$, then because $|j|\left\|q_{n+2} \theta\right\|<q_{n+1}\left\|q_{n+2} \theta\right\|<q_{n+1} / q_{n+3}<\frac{1}{2}$, it must be by (17) that $|j|\left\|q_{n+2} \theta\right\|<\lambda \varepsilon$. But, since $q_{n}<|j|$, we would also have $c^{3}<q_{n}\left\|q_{n+2} \theta\right\|<\lambda \varepsilon<c^{3}$ by (20). Therefore, there exists $i,|i|<q_{n+2}$, with $\|i \theta-t\|=z_{n+2}, i \neq j$. By the triangle inequality, $\|(i-j) \theta\|<\varepsilon_{n+1}+\varepsilon_{n+2}$, and so $q_{n+1}\|(i-j) \theta\|<\varepsilon$. On the other hand $|i-j|<q_{n+1}$ $+q_{n+2}=q_{n+3}$, so $\|(i-j) \theta\|>\left\|q_{n+3} \theta\right\|$. We have again from (20) $c^{3}<q_{n+1}\left\|q_{n+3} \theta\right\|<\varepsilon$, a contradiction. The theorem is proved.

REMARK. Equations (11) and (17) serve as substitutes for equations (27) and (38) of [7]. Lemmas 2-11 of [7] use these equations only, and therefore one can conclude from Lemma 11 that if $(10)$ is true, then $t$ is expandable in an infinite series $t=m \theta+\sum_{n=1}^{\infty} b_{n} q_{n} \theta, b_{n} \in \boldsymbol{Z}$, convergent in $X$ with the property $\lim _{n \rightarrow \infty} b_{n} q_{n}\left\|q_{n} \theta\right\|$ $=0$. This serves as a second proof of Theorem 2 of course, but we are mentioning it because it is proved in Lemma 14 and the Remark, p. 33, of [7] that no nonzero number $t$ can be so represented. Finally, it is possible to improve Lemma 12 of [7] to obtain from (10) that $\lim _{n \rightarrow \infty} e\left(b_{n} \alpha\right)=1$, where $b_{n}$ are as above in the representation of $t$. The details are rather cumbersome, and so we omit them. Notice however that if $b_{n} \neq 0$ infinitely often, then by the Riemann-Lebesgue lemma the set of $\alpha$ for which $\lim _{n \rightarrow \infty} e\left(b_{n} \alpha\right)=1$ has measure 0 . We conclude that for fixed $t$ and almost all $\alpha$ the sequence $q_{1} \alpha, q_{2} \alpha, \ldots$ is well distributed modulo 1 , where $S(\theta, I)$ $=q_{1}, q_{2}, \ldots$ and $I$ has length $t$. (Note the reversion to the notation of the introduction for $q_{n}$.) There exist sequences $q_{1}, q_{2}, \ldots$ of integers with bounded gaps such that $q_{n} \alpha$ is well distributed for no value of $\alpha$ [10].

4. Remarks. One consequence of Theorem 1 is that well distributed integer sequences can grow as fast as polynomials. We do not know if there is one that grows 
faster than any polynomial, but we expect there will be one. On the other hand it is not possible for a lacunary sequence $q_{1}, q_{2}, \ldots, q_{n+1} / q_{n} \geqq \lambda>1$, to be uniformly distributed. In fact, the set of $\alpha$ for which the limit (1) fails to exist will have Hausdorff dimension 1 [2].

\section{REFERENCES}

1. J. W. S. Cassels, Global fields, Proc. Instruc. Conf. Algebraic Number Theory (Brighton, 1965), Thompson, Washington, D. C., 1967, pp. 42-84. MR 36 \#5106.

2. P. Erdös and S. J. Taylor, On the set of points of convergence of a lacunary trigonometric series and the equidistribution properties of related sequences, Proc. London Math. Soc. (3) 7 (1957), 598-615. MR 19, 1050.

3. G. H. Hardy and E. M. Wright, On introduction to the theory of numbers, Clarendon Press, Oxford, 1962.

4. H. Heilbronn, Zeta-functions and L-functions, Proc. Instruc. Conf. Algebraic Number Theory (Brighton, 1965), Thompson, Washington, D. C., 1967, pp. 204-230. MR 36 \#1414.

5. I. Niven, Uniform distribution of sequences of integers, Trans. Amer. Math. Soc. 98 (1961), 52-61. MR $22 \# 10971$.

6. W. A. Veech, Some questions of uniform distribution, Ann. of Math. (to appear).

7. - Strict ergodicity in zero dimensional dynamical systems and the Kronecker-Weyl theorem mod 2, Trans. Amer. Math. Soc. 140 (1969), 1-33. MR 39 \#1410.

8. E. Weiss, Algebraic number theory, McGraw-Hill, New York, 1963. MR 28 \#3021.

9. H. Weyl, Uber die Gleichverteilung von Zahlen mod E ins, Math. Ann. 77 (1916), 313-352.

10. A. Zame, On the measure of well-distributed sequences, Proc. Amer. Math. Soc. 18 (1967), 575-579. MR 35 \#4172.

Rice University, Houston, TeXas 77001 